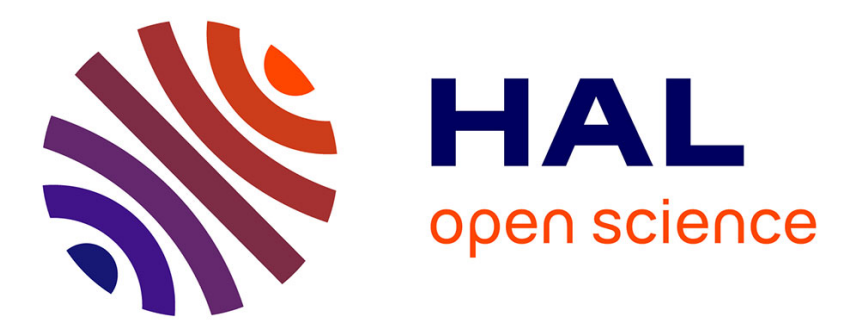

\title{
Backward optical parametric efficiency in quasi-phase-matched GaN waveguide presenting stitching faults
}

C. Montes, Pierre Aschieri, Marc de Micheli

\section{- To cite this version:}

C. Montes, Pierre Aschieri, Marc de Micheli. Backward optical parametric efficiency in quasi-phasematched GaN waveguide presenting stitching faults. Optics Letters, 2013, 38 (12), pp.2083-2085. hal-00832218

\section{HAL Id: hal-00832218 \\ https://hal.science/hal-00832218}

Submitted on 10 Jun 2013

HAL is a multi-disciplinary open access archive for the deposit and dissemination of scientific research documents, whether they are published or not. The documents may come from teaching and research institutions in France or abroad, or from public or private research centers.
L'archive ouverte pluridisciplinaire HAL, est destinée au dépôt et à la diffusion de documents scientifiques de niveau recherche, publiés ou non, émanant des établissements d'enseignement et de recherche français ou étrangers, des laboratoires publics ou privés. 


\title{
Backward optical parametric efficiency in quasi-phase-matched GaN waveguide presenting stitching faults
}

\author{
Carlos Montes*, Pierre Aschieri, and Marc de Micheli \\ Laboratoire de Physique de la Matière Condensée (CNRS UMR 7336), Université de Nice-Sophia Antipolis, Parc Valrose, \\ F-06108 Nice Cedex2, France \\ *Corresponding author: carlos.montes@unice.fr
}

Compiled May 26, 2013

\begin{abstract}
We model a backward mirrorless optical parametric oscillator in a fragmented GaN waveguide consisting in a sequence of submicronic periodically poled elements separated by uniformly polarized connection sections representing stitching errors. We find that the generated coherent phase of the backscattered wave locks the phases of the forward propagating waves in such a way that the dynamics is nonintuitivelly as much efficient as for a uniform quasi phase matched waveguide. The best coherence transfer to the backward wave, obtained for perfect group velocity matching of the forward propagating waves, requires a nanoscale poled periodicity which is achieved in GaN through epitaxy.
\end{abstract}

In periodically poled (PP) optical nonlinear quadratic materials, it is possible to choose a submicronic poling period such that the phase-matched signal and idler waves are generated in opposite directions, therefore establishing a distributed feedback mechanism, which enables optical parametric oscillation without the need of external mirrors or surface coatings. The mirrorless optical parametric oscillator (MOPO), employing this mechanism, was theoretically studied from $1966[1,2]$ and experimentally realized in a $\mathrm{PP} \mathrm{KTiOPO} 4$ (PPKTP) crystal with $800 \mathrm{~nm}$ periodicity as a quasi-phase-matched (QPM) nonlinear medium [3]. One of the remarkable properties of this backward MOPO is the strong asymmetry in spectral bandwidth between the signal and the idler pulses: the bandwidth of the forward signal is comparable to the pump bandwidth, whereas that of the backward idler is typically one to two orders of magnitude narrower [4]. This has been recently demonstrated both experimentally and numerically in the PPKTP MOPO when the pump wave exhibits deterministic phase modulation for relatively narrowband $(1.2 \mathrm{THz})$ linearly-chirped pump pulses [5] and relatively broadband $(4 \mathrm{THz})$ pump pulses too [6]. In accordance with the convection-induced phase-locking mechanism [7-11], the phase modulation of the pump is coherently transferred to the forward parametric wave, while the backward wave retains a narrow bandwidth and high coherence.

In this Letter we study this MOPO process in a GaN waveguide configuration, and we show that the mirrorless optical parametric oscillation takes place even in the presence of stitching errors, which are very likely to occur during the fabrication process of the waveguide. Indeed, the realization of centimeter long devices presenting a submicrometer periodicity is not possible without moving the sample under the writing beam. This displacement introduces the stitching faults. The device is then formed by a sequence of PP poled elements of about 200 $\mu \mathrm{m}$ long (PPGaN bricks) separated by uniformly polar- ized connection sections of several micrometers long representing the stitching errors. The parameters used in the model are those of a periodically poled GaN waveguide (PPGaN) grown by epitaxy on sapphire substrates [12] and presenting submicronic periodicity of the $\chi^{(2)}$ coefficient [13]. In this material, the reasonably large value of the second order susceptibility $\left(\chi_{33}^{(2)}=10.6 \mathrm{pm} / \mathrm{V}\right.$ measured in [14]) opens the door for parametric counterpropagating oscillation. It is found that the generated coherent phase of the backscattered wave locks the phases of the forward propagating waves in such a way that the dynamics is almost insensitive to the short links between the PP elements where quasi-phase-matching is not preserved. Nonintuitivelly, we obtain as much efficiecy as for a uniform quasi phase matched waveguide.

In a MOPO, the pump wave is down-converted into forward-propagating and backward-propagating parametric waves, in accordance with energy conservation, $\omega_{p}=\omega_{f}+\omega_{b}$ and counterpropagating quasi-phase matching, $k_{p}-k_{f}+k_{b}=K_{G}$, where $j=p, f, b$ denote the pump, the forward and the backward parametric waves, respectively. $K_{G}=2 \pi / \Lambda_{G}$ is the grating vector given by $\Lambda_{G}$, the modulation period of the second-order nonlinear coefficient. The effect of coherence transfer effect to the backward wave is especially pronounced when the pump wavelength and the modulation period of the nonlinear medium are chosen so that the group velocities of the pump and the forward wave are exactly matched [8]. This may be achieved in a GaN guided geometry with a group velocity dispersion modified by a particular profile of the refractive index, a technique widely used in fiber to compensate the material dispersion with the waveguide dispersion [15]. Then it is possible to match the pump and the idler group velocities exactly and the counterpropagating wave will be the signal. This requires a short PP period $\Lambda_{G}$ of hundreds of nm in length which may be realized in the GaN structure thanks to epitaxy $[13,14]$. For $z$-polarized waves in PPGaN, matching the group velocities can be achieved by designing the experiment 
so that the pump and the forward wave are on different sides of the maximum on the group-velocity curve shown in Fig. 1. This shape of group-velocity curve may be obtained in the waveguide PPGaN structure composed of a strip-loaded GaN of $2 \mu \mathrm{m}$ width and $1.8 \mu \mathrm{m}$ depth on a AlInN buffer which has been realized on Sapphire substrate by epitaxy. The Sellmeier relation given in [14] is used for material dispersion and finite waveguide dispersion corrections [15] yield the corresponding group velocity curve of Fig. 1.

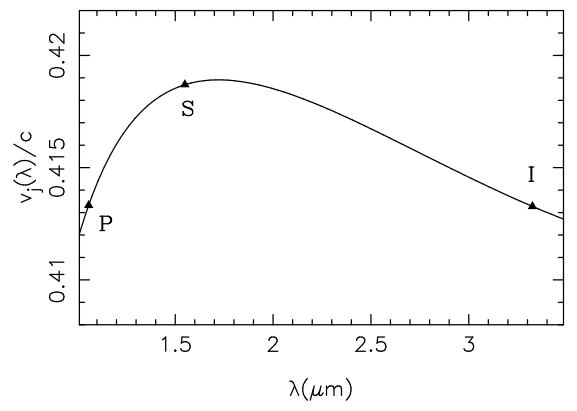

Fig. 1. Group velocity for the $z$-polarized waves in GaN as function of the wavelength, calculated from the Sellmeier expansions in [14] with a particular profile of the refractive index of the guide [15]. The triangles mark the configuration of perfectely matched pump and idler group velocities $\left(\left|\mathrm{v}_{\mathrm{p}}-\mathrm{v}_{\mathrm{i}}\right|=0\right)$, and are labeled by a $\mathbf{P}$ for the pump, a $\mathbf{S}$ for the signal, and an $\mathbf{I}$ for the idler.

The pump intensity required to reach threshold for MOPO operation, $I_{p, t h}$, can be estimated from the plane-wave monochromatic model for the undepleted pump parametric approximation [2] as $I_{p, t h}=$ $c \varepsilon_{0} n_{p} n_{f} n_{b} \lambda_{f} \lambda_{b} / 2 L^{2} d_{e f f}^{2}$, where $c$ is the speed of light in vacuum, $\varepsilon_{0}$ is the permittivity of free space, $n_{j}$ denotes the refractive indices, $\lambda_{j}$ denotes the backward and forward wavelengths, $L$ is the nonlinear interaction length and $d_{\text {eff }}=7 \mathrm{pm} / \mathrm{V}$ is the effective quadratic nonlinearity.

Numerical simulations of the nonlinear three-wave interactions in a MOPO solve the coupled wave equations in the slowly-varying envelope approximation. The field amplitudes, $\mathbf{A}_{\mathbf{j}}(\mathbf{j}=\mathbf{p}, \mathbf{s}, \mathbf{i})$, of the pump and the parametric backward signal wave and forward idler wave, evolve with the coupled equations:

$$
\begin{aligned}
\left(\partial_{t}+\mathrm{v}_{\mathrm{p}} \partial_{x}+\gamma_{p}+i \beta_{p} \partial_{t t}\right) \mathbf{A}_{\mathbf{p}} & =-\sigma_{p}(x) \mathbf{A}_{\mathbf{s}} \mathbf{A}_{\mathbf{i}} \\
\left(\partial_{t}-\mathrm{v}_{\mathrm{s}} \partial_{x}+\gamma_{s}+i \beta_{s} \partial_{t t}\right) \mathbf{A}_{\mathbf{s}} & =\sigma_{s}(x) \mathbf{A}_{\mathbf{p}} \mathbf{A}_{\mathbf{i}}^{*} \\
\left(\partial_{t}+\mathrm{v}_{\mathrm{i}} \partial_{x}+\gamma_{i}+i \beta_{i} \partial_{t t}\right) \mathbf{A}_{\mathbf{i}} & =\sigma_{i}(x) \mathbf{A}_{\mathbf{p}} \mathbf{A}_{\mathbf{s}}^{*}
\end{aligned}
$$

where $\sigma_{j}(x)=2 \pi d_{e f f} \mathrm{v}_{\mathrm{j}} / \lambda_{j} n_{j}$ are the nonlinear coupling coefficients in the PP elements, while $\sigma_{j}(x)=0$ within the uniformly polarized gaps, $\gamma_{j}$ and $\beta_{j} \equiv \mathrm{v}_{\mathrm{j}} \beta_{2, j} / 2$ are the damping and dispersion coefficients. The input parameters in the model are the properties of the nonlinear medium and the pump amplitude at the input face, $\mathbf{A}_{\mathbf{p}}(x=0, t)$, generating outputs of $\mathbf{A}_{\mathbf{p}}(x=L, t)$, $\mathbf{A}_{\mathbf{i}}(x=L, t)$ and $\mathbf{A}_{\mathbf{s}}(x=0, t)$. The nonlinear counterpropagation dynamics in the quadratic MOPO in the presence of group-velocity dispersion, which introduces second-order time derivatives, makes use of a numerical scheme which combines the trajectories method, extensively used for the treatment of stimulated Brillouin back-scattering problems [16], with fast Fourier transformation (FFT) to account for the group-velocity dispersion effects in the spectral domain $[6,17]$.

In all simulations, the nonlinear medium is a GaN structured ensemble composed by a train of $\mathrm{N}$ periodically-poled GaN elements of length $\ell$ disposed in a straight row as shows Fig. 2. Simulations for this seg-

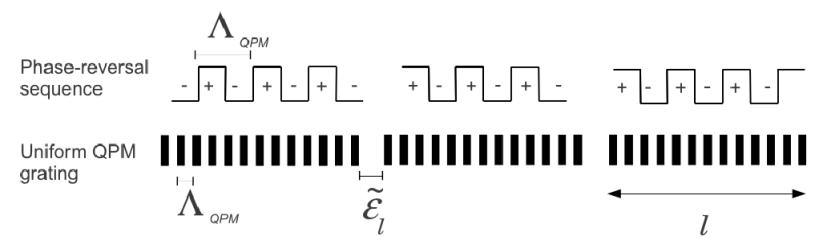

Fig. 2. The quasi-phase-matching (QPM) is obtained by a phase-reversal sequence of the ferroelectric domains with a grating periodicity $\Lambda_{Q P M}$. The segmented backward MOPO is composed by a train of periodically poled GaN elements $\ell$ separated by uniformly polarized connection sections $\tilde{\varepsilon_{\ell}}$ representing stitching errors.

mented structure of length $\mathrm{L}_{\text {seg }}=\mathrm{N} \ell+(\mathrm{N}-1) \tilde{\varepsilon}_{\ell}$, where $\tilde{\varepsilon_{\ell}}$ is the short random length of the gaps linking the PPGaN bricks, will be compared to the case of a continuos medium of length $\mathrm{L}=\mathrm{N} \ell$. For $\mathrm{L}=64 \ell=1.25 \mathrm{~cm}$ each element has a length $\ell=195 \mu \mathrm{m}$. The segmented MOPO will have a length $\mathrm{L}_{\text {seg }}=1.32 \mathrm{~cm}$ with 63 random gaps $\tilde{\varepsilon}_{\ell}=\left\langle\tilde{\varepsilon}_{\ell}\right\rangle+\delta \varepsilon_{\ell}$ where $\delta \varepsilon_{\ell}$ is random uniformly distributed in the interval $\left[-\Delta \varepsilon_{\ell},+\Delta \varepsilon_{\ell}\right]$ with $\left\langle\tilde{\varepsilon}_{\ell}\right\rangle=10.8 \mu \mathrm{m}$ for the mean value and $\Delta \varepsilon_{\ell} /\left\langle\tilde{\varepsilon}_{\ell}\right\rangle=0.15$. The waveguide has a section $\mathrm{S}_{\text {eff }}=3.6 \mu \mathrm{m}^{2}$.

We choose the three QPM waves marked on the Fig. 1, and we model MOPO operation with a broadband stochastic pump pulse with a FWHM temporal length of $100 \mathrm{ps}$, and where the spectral FWHM width is $\Delta \nu_{p}=4.40 \mathrm{THz}\left(\Delta \lambda_{p}=15 \mathrm{~nm}\right)$, centered at $\lambda_{\mathrm{p}}=1.06 \mu \mathrm{m} \quad$ with $\quad \mathrm{n}_{\mathrm{p}}=2.30856, \quad \mathrm{v}_{\mathrm{p}} / \mathrm{c}=0.41337$, $\beta_{2, \mathrm{p}}=0.286 \mathrm{ps}^{2} / \mathrm{m} ; \quad$ and with $\lambda_{\mathrm{s}}=1.56242 \mu \mathrm{m}$, $\mathrm{n}_{\mathrm{s}}=2.26580, \quad \mathrm{v}_{\mathrm{s}} / \mathrm{c}=0.41872, \quad \beta_{2, \mathrm{~s}}=0.0647 \mathrm{ps}^{2} / \mathrm{m}$; $\lambda_{\mathrm{i}}=3.29791 \mu \mathrm{m}, \quad \mathrm{n}_{\mathrm{i}}=2.12353, \quad \mathrm{v}_{\mathrm{i}} / \mathrm{c}=0.41337$, $\beta_{2, \mathrm{i}}=-0.418 \mathrm{ps}^{2} / \mathrm{m}$. The $\mathrm{QPM}$ period $\Lambda_{G}=335$ $\mathrm{nm}$, may be realized in GaN by epitaxy [14].

The pump pulse is issued from a Gaussian stochastic process translationally invariant with zero mean $\left\langle\mathbf{A}_{\mathbf{p}}(\mathbf{x}=\mathbf{0}, \mathbf{t})\right\rangle$ and exponential autocorrelation function $\left\langle\mathbf{A}_{\mathbf{p}}\left(x=0, t^{\prime}+t\right) \mathbf{A}_{\mathbf{p}}^{*}(x=0, t)\right\rangle=\left|\mathbf{A}_{\mathbf{p}}^{\mathbf{0}}\right|^{2} \exp \left(-|t| / \tau_{c}\right)$, where $\tau_{c}=1 / \pi \Delta \nu_{p}$ is the correlation time. Note that, while the pump phase fluctuations are absorbed by the idler wave thanks to the phase-locking mechanism, the generation of the signal still remains affected by the intensity fluctuations of the pump. In the presence of small group velocity difference between the pump and the signal, which is the case for the forward OPOs, pump inten- 

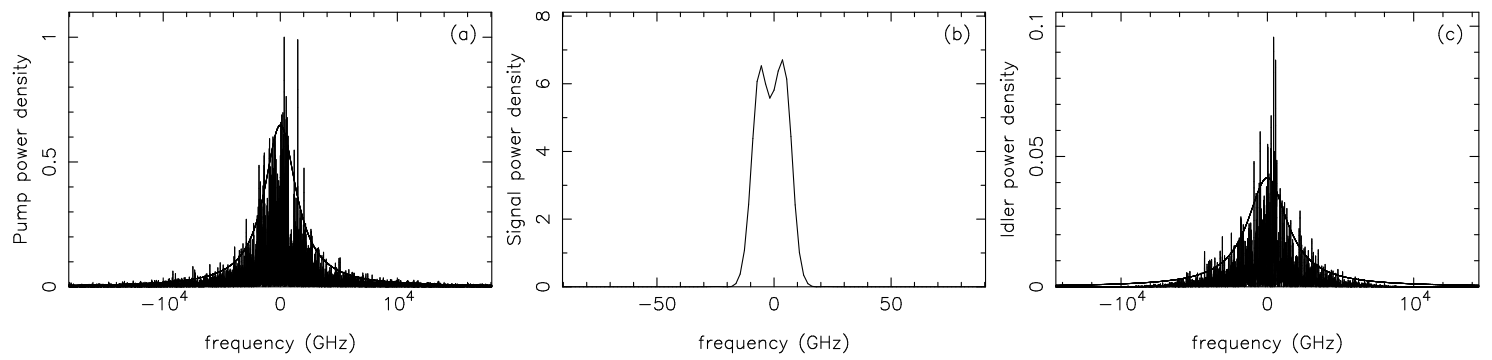

Fig. 3. Stochastic pumped MOPO (segmented in 64 elements): (a) Incoherent pump spectrum fitted by a Gaussian envelope of FWHM $\Delta \nu_{p}(\mathrm{~L})=4.40 \mathrm{THz}$, (b) backward signal spectrum with $\Delta \nu_{s}(0)=18.18 \mathrm{GHz}$, and (c) forward idler spectrum with a Gaussian fitting of FWHM $\Delta \nu_{i}(\mathrm{~L})=3.31 \mathrm{THz}$. The coherence gain attains $\Delta \nu_{p} / \Delta \nu_{s}=242$.

sity fluctuations are partly transferred to the signal component $[9,11]$. Conversely, in the presence of the strong group velocity difference of the backward OPO, intensity fluctuations of the pump are averaged by the strong convection effect, which thus leads to an enhancement of the coherence of the backward signal [6]. The higher the incoherence of the pump, the larger the amount of coherence transfer as studied in section V.B of Ref. [8].

Stochastic MOPO operation in both continuous and segmented structures are simulated with a pump pulse of $\mathrm{I}_{p}=2 \mathrm{GW} / \mathrm{cm}^{2}$ intensity, the MOPO threshold intensity being $\mathrm{I}_{p, t h}=0.937 \mathrm{GW} / \mathrm{cm}^{2}$. The peak power yields $72 \mathrm{~W}$, a pulsed energy of $7.2 \mathrm{~nJ}$, and a fluence of $0.2 \mathrm{~J} / \mathrm{cm}^{2}$ which remains below the damage threshold for GaN [18]. The pump and the parametric spectra are illustrated in Fig 3, showing a backward signal with a spectral width of $\Delta \nu_{s}=18.18 \mathrm{GHz}$, still narrow compared to the widths of the depleted pump, $\Delta \nu_{p}(L)=$ $4.4 \mathrm{THz}$, and the forward idler, $\Delta \nu_{i}=3.31 \mathrm{THz}$. By integrating the spectra, it is found that the conversion into parametric waves is $I_{s}(0) / I_{p}(0)=0.1190 \pm 0.0004$ for the signal and $I_{i}(L) / I_{p}(0)=0.0594 \pm 0.0002$ for the idler, while the pump depletion yields $1-I_{p}(L) / I_{p}(0)=$ $0.1700 \pm 0.0006$. Nonintuitivelly, the stitched MOPO is as efficient as the uniform quasi phase matched waveguide MOPO. Its efficiency turns to be of $92.7( \pm 0.4) \%$ with respect to the latter and the coherence transfer attains $\Delta \nu_{p}(L) / \Delta \nu_{s}(0)=242$. The phase-locking behaviour inside the PPGaN bricks is like that illustrated in Fig.4(b) of Ref. [6] for a chirped pump. To avoid other nonlinearities, such as stimulated Raman scattering, which is the most harmful nonlinearity competing with the parametric downconversion [3], we can further decrease the pump intensity. With $\mathrm{I}_{p}=1.5 \mathrm{GW} / \mathrm{cm}^{2}$ and a fluence of 0.15 $\mathrm{J} / \mathrm{cm}^{2}$, we obtain $15 \%$ pump depletion and a coherence gain $\Delta \nu_{p}(L) / \Delta \nu_{s}(0)=173$.

In summary, we have numerically simulated a MOPO in realistic PPGaN waveguides by using stochastic pump pulses of up to $4.4 \mathrm{THz}$ bandwidth. It has been shown that mirrorless optical parametric oscillation occurs with reasonable pump power and that the coherence transfer attains 242. Parametric three-wave interaction is a reversible process and downconversion in one $\mathrm{PP}$ element could be destroyed in the adjacent one if the phase shift produced in the gap reverses the downconversion, as is the case for a standard forward OPO. Quite surprisingly in the backward MOPO, the structure presenting stitching errors is almost as efficient as a perfect one, like that presented in [6], because the coherent phase of the backward wave locks the phases in such a way that the dynamics is almost insensitive to the random length of the uniformly polarized junctions.

\section{References}

1. S. E. Harris, Appl. Phys. Lett. 9, 114-116 (1966).

2. Y.I. Ding, J.B. Khurgin, IEEE J. Quantum Electron. 32, 1574-1562 (1996).

3. C. Canalias, V. Pasiskevicius, Nature Photon. 1, 459-462 (2007).

4. V. Pasiskevicius, C. Canalias, G. Strömqvist, F. Laurell, SPIE Proc. 6875, 6875081-12 (2008).

5. G. Strömqvist, V. Pasiskevicius, C. Canalias and C. Montes, Phys. Rev. A 84, 023825 (2011).

6. G. Strömqvist, V. Pasiskevicius, C. Canalias, P. Aschieri, A. Picozzi, and C. Montes, J. Opt. Soc. Am. B 29, 1194-1202 (2012).

7. A. Picozzi and M. Hælterman, Phys. Rev. Lett. 86, 2010 (2001).

8. A. Picozzi, C. Montes, M. Hælterman, Phys. Rev. E 66, 056605-1-14 (2002).

9. C. Montes, A. Picozzi, K. Gallo, Opt. Commun. 237, 437-449 (2004).

10. A. Picozzi and P. Aschieri, Phys. Rev. E 72, 046606-1-12 (2005).

11. C. Montes, W. Grundkötter, H. Suche, and W. Sohler, J. Opt. Soc. Am. B 24, 2796-2806 (2007).

12. A. Chowdhury, H. M. Ng, M. Bhardwaj, and N. G.Weimann, Appl. Phys. Lett. 83, 1077-1079 (2003)

13. S. Pezzagna, P. Vennéguès, N. Grandjean, A. D. Wieck, and J. Massies, Appl. Phys. Lett. 87, 062106 (2005).

14. S. Pezzagna, J. Brault, M. Leroux, J. Massies, and M. de Micheli, J. Appl. Phys. 103, 123112-1-7 (2008).

15. M. Monerie, IEEE J. Quantum Electron. 18, 535 (1982).

16. C. Montes, A. Mikhailov, A. Picozzi and F. Ginovart, Phys. Rev. E 55, 1086-1091 (1997).

17. C. Montes, P. Aschieri, and A. Picozzi, SPIE Proc. 8011, 801136-1-10 (2011).

18. X.C. Wang, G.C. Lim, F.L. Ng, W. Liu, and S.J. Chua, Applied Surface Science 252, 14921497 (2005). 


\section{References}

ence 252, 14921497 (2005).

1. S. E. Harris, "Proposed backward wave oscillation in the infrared", Appl. Phys. Lett. 9, 114-116 (1966).

2. Y.I. Ding, J.B. Khurgin, "Backward optical parametric oscillators and amplifiers", IEEE J. Quantum Electron. 32, 1574-1562 (1996).

3. C. Canalias, V. Pasiskevicius, Nature Photon. "Mirrorless optical parametric oscillator", 1, 459-462 (2007).

4. V. Pasiskevicius, C. Canalias, G. Strömqvist, F. Laurell, "Mirrorless OPO: first steps towards unlocking the potential of counter-propagating three-wave interactions" (invited), SPIE Proc. 6875, 6875081-12 (2008).

5. G. Strömqvist, V. Pasiskevicius, C. Canalias and C. Montes, "Coherent phase-modulation transfer in counterpropagating parametric down-conversion", Phys. Rev. A 84, 023825 (2011).

6. G. Strömqvist, V. Pasiskevicius, C. Canalias, P. Aschieri, A. Picozzi, and C. Montes, "Temporal coherence in mirrorless optical parametric oscillators", J. Opt. Soc. Am. B 29, 1194-1202 (2012).

7. A. Picozzi and M. Hælterman, "Parametric Three-Wave Soliton Generated from Incoherent Light", Phys. Rev. Lett. 86, 2010 (2001).

8. A. Picozzi, C. Montes, M. Hælterman, "Coherence properties of the parametric three-wave interaction driven from an incoherent pump", Phys. Rev. E 66, 056605-114 (2002).

9. C. Montes, A. Picozzi, K. Gallo, "Ultra-coherent output from an incoherent cw-pumped singly resonant optical parametric oscillator", Opt. Commun. 237, 437-449 (2004).

10. A. Picozzi and P. Aschieri, "Influence of dispersion on the resonant interaction between three incoherent waves", Phys. Rev. E 72, 046606-1-12 (2005).

11. C. Montes, W. Grundkötter, H. Suche, and W. Sohler, "Coherent signal from incoherently cw-pumped singly resonant Ti:LiNbO3 integrated optical parametric oscillators", J. Opt. Soc. Am. B 24, 2796-2806 (2007).

12. A. Chowdhury, H. M. Ng, M. Bhardwaj, and N. G.Weimann, "Second-harmonic generation in periodically poled GaN", Appl. Phys. Lett. 83, 1077-1079 (2003)

13. S. Pezzagna, P. Vennéguès, N. Grandjean, A. D. Wieck, and J. Massies, "Submicron periodic poling and chemical patterning of GaN", Appl. Phys. Lett. 87, 062106 (2005).

14. S. Pezzagna, J. Brault, M. Leroux, J. Massies, and M. de Micheli, "Refractive indices and elasto-optic coefficients of GaN studied by optical waveguiding", J. Appl. Phys. 103, 123112-1-7 (2008).

15. M. Monerie, "Propagation in doubly clad single-mode fibers" IEEE J. Quantum Electron. 18, 535 (1982).

16. C. Montes, A. Mikhailov, A. Picozzi and F. Ginovart, "Dissipative three-wave structures in stimulated backscattering. I. A subluminous solitary attractor", Phys. Rev. E 55, 1086-1091 (1997).

17. C. Montes, P. Aschieri, and A. Picozzi, "Model for Coherence Transfer in a Backward Optical Parametric Oscillator", SPIE Proc. 8011, 801136-1-10 (2011).

18. X.C. Wang, G.C. Lim, F.L. Ng, W. Liu, and S.J. Chua, "Femtosecond pulsed laser-induced periodic surface structures on GaN/sapphire", Applied Surface Sci- 